\title{
Engineering the Next Generation of Large Area Displays: Prospects and Pitfalls
}

\author{
J. D. Carey \\ Advanced Technology Institute, School of Electronics and Physical Sciences, \\ University of Surrey, Guildford, GU2 7XH
}

Considerable effort is currently been expounded into the development and improvement of the myriad of display technologies that have come to the market place. In this paper several key questions are addressed in the development of the future generation of large area field emissionbased displays based upon semiconducting amorphous carbon thin films and carbon nanotubes. The development of carbon based cathodes has to date proceeded along empirical lines with attempts to correlate the variation of field emission characteristics with changes in deposition or post deposition processing parameters, often without a full explanation being forthcoming. In addition, there have been incidents of incorrect interpretation of some of the results due to a lack of an appreciation of the significant differences between the different types of amorphous carbon film that exist. It is only recently that a fuller understanding of the different electron emission mechanisms has begun to emerge through an understanding of the roles played by the electrical and structural inhomogeneity at a nanometer level. This 'intrinsic dielectric inhomogeneity' is shown to possess some remarkable electronic properties which also has important consequences for extrinsic inhomogeneous nanometer systems such as carbon nanotube (CNT) and CNT-polymer composite based displays. The future outlook for broad area displays based upon amorphous carbon and carbon nanotubes is also addressed. 


\section{Introduction}

The growing trend in information exchange has manifested itself in the continuous development of different display technologies with improvements in the traditional cathode ray tube and in liquid crystals based displays being made as well as the emergence of plasma displays, organic polymer displays and field emission displays (FEDs). The type and characteristics of the information that is to be portrayed often determines the technology that is to be employed. For example, high resolution over small screens is needed for most laptop computers but screens used in sporting stadia need not possess such high resolution, however, the information must be able to be disseminated over large areas. Figure 1 broadly categorises displays into projection, off screen and direct view, the final category being conveniently sub-divided into cathode ray tube (CRT) and flat panel display (FPD) technology. Further sub-division is also possible where a useful distinction between emitter-based FPDs, such as field emission displays (FEDs) and organic light emitting diodes (OLEDs) on one hand and non-emitter-based FPDs, such as active matrix liquid crystal displays (AMLCDs) is possible. In order to quantify the importance that displays play, market forecasts predict that the FPD market will be worth $\$ 70$ billion by the year 2010 and that by 2007 thin film transistor based LCD production will have exceeded CRT production (Funaki and Mochizuki, 1999). Whilst the dominant technologies associated with CRTs and FPDs are extremely mature and are continually improving in terms of viewing angle and power consumption, there remain well established problems with the scaling up of these technologies to larger screen diagonals.

Figure 2 shows how the different types of both existing and emerging technology vary for different sized screen diagonals. It can be seen that in the 50-100 cm diagonal region that the two dominant existing technologies are CRTs and plasma displays panels (PDPs) (Burden, 2001a). In a CRT display, as the diagonal size increases the angle by which the electron beam is required to be deflected also increases. As the maximum deflection angle in a CRT is limited, it is necessary to 
increase the tube length and hence the size and mass of large diagonal CRTs. This, in turn, leads to bulky displays, which are costly to manufacture. For PDPs the high cost brought about by expensive electronics, the cost of suitably large substrates and problems striking and maintaining a plasma are more problematic for $50-100 \mathrm{~cm}$ diagonals. For this size diagonal the emerging technologies are FEDs and OLEDs. OLED based materials and displays have been reviewed previously in this series (Samuel, 2000) and in this article we will concentrate on FEDs.

In contrast to single electron sources, such as the CRT, FEDs are based on the extraction of electrons under the action of an applied electric field from multiple sources. This can be in the form of the so-called 'Spindt tip' arrays (Fig. 3(a)) consisting of sharp metal, often Mo or W, or Si tips together with a gate electrode and insulator and are prepared using lithographic techniques. These tip arrays can suffer from tip poisoning due the presence of residual gas molecules that will degrade overall device performance. In addition, since 'Spindt tip' technology is a lithographic based process there are difficulties in scaling up the production process e.g. large volume metal evaporators are required to ensure that the metal atoms are normally incident onto the substrate in order to achieve good uniformity. From these tip based emitters, field emission (FE) is largely determined by the geometric shape of the emitter due to a (geometric) enhancement of the applied electric field. FE from a range of 'flat' materials such as CVD diamond and amorphous semiconducting thin films has also been observed (Silva et al. 2002a). Early interest in amorphous carbon $(a-C)$ films as cold cathodes was driven by the hope that these films possessed negative electron affinity similar to that found on certain $\mathrm{H}$ terminated diamond surfaces. This meant that for an $a$-C film to act as a cathode all that was needed was to ensure that there was an efficient supply of electrons injected into the conduction band of the material, and that alone allow for a suitable emission current at the front surface. Both types of carbon cathode material can be deposited over large areas but the latter has the added advantage that it can be deposited at low temperatures without the need for complex and expensive lithography. A-C films also have the attractive feature 
Published in Phil. Trans. Roy. Soc. A 361, 2891 (2003)

that by varying the deposition conditions a wide range of different type of film can be produced -

Fig. 3(b). Before discussing how these material systems can be used in broad area displays it is worth exploring why these materials can be useful as electron sources. It is worth bearing in mind that apart from the deposition and/or lithography issues discussed above, from a technological view point a low threshold field with good emission site density is required and for a practical display a current density of $1-10 \mathrm{~mA} \mathrm{~cm}^{-2}$ is needed. It is also worth stating that efficient electron emission is also required for microwave amplifiers, where powers in excessive of $20 \mathrm{~W}$ are possible. Integrating field emission arrays into klystrons or traveling wave tubes higher operating frequencies in excessive of $100 \mathrm{GHz}$ are possible have been reported (Zhu, 2001).

\section{Electron Field Emission: Theory, Materials and Mechanisms}

Electron emission from metal surfaces was first reported by Wood in 1897 (Wood, 1897). The emission that takes place from a metal surface is principally determined by the strength of the applied field in combination with the magnitude of the work function as shown in Figure 4. In general, as the electric field is increased first thermionic emission over the top of the surface barrier (work function) will occur, followed by emission over the field reduced barrier (Schottky emission). Emission will then occur partly over the barrier if the temperature is high enough and partly through the barrier. As the temperature is lowered emission will come from electrons tunnelling through the barrier (Fowler-Nordheim tunnelling) from electron states at the Fermi level and finally at very high fields, emission from states below the Fermi level (ballistic electron emission). Throughout the long history of field emission the seminal theory of Fowler and Nordheim played, and continues to play, a central role as the conventional explanation of emission (Fowler and Nordheim, 1928). The FN theory has been applied to explain the emission from gated emitter arrays, CVD grown diamond films with grain boundaries, atomically smooth amorphous carbon thin films and carbon nanotubes, both single and multiwall, and as such its application to these structures is somewhat 
remarkable when considering the assumptions on which the theory is based (Zhu, 2001). The standard FN theory is based on the following assumptions:

(i) the emitter is an atomically smooth and clean metal surface,

(ii) the metal has a free electron band structure and the electrons in the metal obey Fermi-Dirac statistics,

(iii) emission occurs at zero Kelvin and the barrier to emission (work function) is uniform and independent of applied electric field,

(iv) the description of the electron tunnelling can be described in terms of the WentzelKramers-Brillouin approximation, and

(v) the barrier to emission can be represented by a triangular potential onto which a classical image potential is superimposed.

Instead of reproducing the derivation of the FN equation based upon assumptions (i) to (v) above, which has been presented in many of the associated textbooks, (Zhu, 2001), it is instructive to examine the final form/s of the equation and discuss the consequences of its application to FE data. Representing the applied electric field by $\mathrm{E}$ and the barrier height by $\Phi$, the emission current $I$ can be expressed as

$$
I=\frac{a A(\beta E)^{2}}{\Phi} \exp \left(\frac{-b \Phi^{3 / 2}}{\beta E}\right)
$$

$a$ and $b$ are constants with values of $1.54 \times 10^{-6} \mathrm{~A} \mathrm{eV} \mathrm{V}^{-2}$ and $6.83 \times 10^{9} \mathrm{eV}^{-3 / 2} \mathrm{Vm}^{-1}$, respectively, and $\mathrm{A}$ is the emission area. $\beta \mathrm{E}$ represents the local electric field and in this context $\beta$ represents the enhancement of the applied electric field. In this formulation $\beta$ is a dimensionless number, however, the FN equation can also be expressed in terms of applied voltage $\mathrm{V}$, which for a diode arrangement with anode-cathode separation $d$, would re-cast equation 1a as 
Published in Phil. Trans. Roy. Soc. A 361, 2891 (2003)

$$
I=\frac{a A\left(\beta^{\prime} V\right)^{2}}{\Phi} \exp \left(\frac{-b \Phi^{3 / 2}}{\beta^{\prime} V}\right)
$$

such that $\beta^{\prime}=\beta / d$. This form of the equation is often seen for gated triodes arrays where $V$ represents the gate voltage. FE measurements are usually performed in either a diode arrangement in which an anode, either a parallel plate or spherical ball electrode is located above a grounded cathode or in a triode arrangement in which a gated electrode is located nearby. The advantage of a spherical anode over a broad area anode is that the latter is prone to misalignment such that one part of the cathode may experiences a different applied field. It is usual to characterise the currentvoltage measurements in terms of the macroscopic field (applied voltage divided by anode-cathode separation) at which the current reaches a prescribed threshold. Regrettably there has been no agreement amongst research groups as to what this value should be and in our research a value of 1 nA has been used. Analysis of field emission $I-V$ characteristics using the Fowler-Nordheim equation is usually performed by plotting $\log \left(I / E^{2}\right)$ against $1 / E$ the slope being $-\mathrm{b} \Phi^{3 / 2} / \beta$. It is not possible from a FE measurement to independently determine both $\beta$ and $\Phi$, though the latter can be determined via other methods. Armed with a knowledge of the work function considerable attention has been paid to significance of the value and origin of the enhancement factor. For example, it can be readily shown that the field enhancement factor associated with a metal hemisphere on a grounded plane is 3 . However, some care must be exercised in the interpretation of enhancement factors since complications arise in comparing theory with experiment. The levels of sophistication that have been developed in understanding that the application of an electric field to a clean metal surface (or other material) are often masked by experimental factors such as the presence of absorbates on the cathode surface. For example, different atoms can induce dipoles on the surface which can change the work function of a metal, the work function itself may possess a dependence on the electric field and/or nanoprotrusions on the surface may act to increase the local electric field. 
Field emission from semiconductors, is also complicated by the possibility of emission from either the conduction or valence bands, from the Fermi level or from surface states. In addition, the effects of band bending, field penetration and internal field emission, particularly from wide gap semiconductors, as well as the possibility of negative electron affinity need to be taken into account. There is also the issue of 'conditioning' of the cathode in which the onset of stable and reproducible emission is only observed after several cycles of the voltage, a process that will be discussed later. Despite these potential complications in understanding the mechanism of emission, the inherently simpler fabrication process and ability to scale up production of thin film emitters have a number of advantages over tip based arrays. Firstly, electron emission from a thin film comes from a much larger area than from a tip array. This results in a lower current density for a given emission current. Secondly, the absence of highly focused electric fields reduces the ion bombardment and sputter erosion of the thin film. These thin film emitters, especially the plethora of amorphous carbon thin films (Silva et al., 2002a) as well as $a$-Si:H films (Tanaka, 1999), have attractive properties for large area electronics. In particular, the ability to deposit different types of $a$-C over large areas at room (or low) temperatures has spurred considerable research into FE from these materials.

By changing the deposition conditions it is possible to change the film properties from wide band gap, low density, high $\mathrm{H}$ containing polymeric $a$-C (PAC) films to higher density, lower gap diamond-like carbon (DLC) films and finally to $\mathrm{sp}^{2}$-rich graphitic-like carbon, as shown in Fig. 3 (b). There is also a non-hydrogen containing film known as tetrahedral amorphous carbon (ta-C) which possess a high film density and a large concentration of paramagnetic defects (Silva et al., 2002a). The electronic structure of $a$-C films can be described in terms of their $\pi$ electrons in mainly $\mathrm{sp}^{2}$ hybridised $\mathrm{C}$ atoms. These $\mathrm{sp}^{2} \mathrm{C}$ atoms are not randomly distributed within the lattice but cluster together either in the form of aromatic rings or olephic chains. Apart from changes to the microstructural properties of the film, it is the changes in the electrical properties of the film that 
are the most important aspect for FE applications. Wide gap $a-\mathrm{C}: \mathrm{H}$ films, deposited using plasma enhanced chemical vapour deposition, possess a low spin density $\left(\sim 10^{17} \mathrm{~cm}^{-3}\right)$ and form a Schottky barrier with the substrate. It has been proposed that the presence of this Schottky barrier at the back contact results in the electrons entering from the Fermi level of the substrate experiencing a high electric field and being accelerated towards the front surface (Amaratunga and Silva, 1996). Such a mechanism is plausible since there is a high degree of field penetration into the film. The field lines from the anode ( 2 terminal) or gate ( 3 terminal) will terminate on the more conductive defects but at a sufficiently low defect density the applied field is not effectively screened.

Three fundamental questions are worthy of addressing before continuing the discussion of the mechanism/s that are responsible for emission from these materials. Firstly, what is the source of the electrons, secondly, how are the electrons transported through the film and finally, how are the electrons emitted into the vacuum. These questions have broadly led to the 'back contact' model of Amaratunga and Silva and later by Forrest and colleagues (Forrest et al., 1998) in which the rate controlling step is the barrier at the film/substrate interface as described above and to the 'front surface/internal enhancement' model, where the transport through the film and the physics of the film surface/vacuum interface are the most important factors (Carey et al., 2000). In order to investigate the transition from the back contact to the front surface a series of $a$-C:H films as a function of deposition self-bias were grown. Changing the deposition self-bias results in changes in the film properties and a transition from PAC to DLC type films. In addition to two terminal FE, measurements of the Tauc optical gap, ESR spin density and ESR peak-to-peak line width were made. The measurement of the Tauc gap gives information about the size of the $\mathrm{sp}^{2} \mathrm{C}$ cluster and the ESR measurements provides information about the concentration and localisation of paramagnetic electron states near to the Fermi level. ESR is sensitive to centers with a net unpaired election spin, for example from odd numbered $\mathrm{sp}^{2} \mathrm{C}$ clusters. The location in the energy gap of $\mathrm{sp}^{2}$ clusters depends on whether they are even or odd numbered and whether they are distorted. 
Undistorted even numbered clusters give rise to states near the Fermi level $\left(\mathrm{E}_{\mathrm{F}}\right)$ only if they are sufficiently large, whereas odd numbered clusters will give rise to states even if composed of a small number of atoms. In general, distorted clusters will give rise to states that are closer to $\mathrm{E}_{\mathrm{F}}$ than undistorted clusters. In this way ESR can give a measure of the density of $\mathrm{sp}^{2}$ states at the Fermi level provided that a majority of these states have a net unpaired electron spin present. Our study of the possible importance of the $\mathrm{sp}^{2}$ phase was instigated following a comparative study of FE with visible Raman spectroscopy of ta-C films (Ilie et al., 2000).

The variation of threshold field, $E_{t h}$, spin density, $N_{s}$, peak-to-peak ESR linewidth, $\Delta B_{p p}$, and Tauc gap for a series of $a-\mathrm{C}: \mathrm{H} / \mathrm{Si}$ films deposited under different self biases is shown in Fig. 5. At a dc self bias of $-50 \mathrm{~V}, \mathrm{E}_{\mathrm{th}}$ is $18 \mathrm{~V} / \mu \mathrm{m}$, which increases to $26 \mathrm{~V} / \mu \mathrm{m}$ at $-90 \mathrm{~V}$ and then decreases at high biases to remain approximately constant at about $12 \mathrm{~V} / \mu \mathrm{m}$. From the lowest bias, $\mathrm{N}_{\mathrm{s}}$ rises rapidly from $3 \times 10^{17} \mathrm{~cm}^{-3}$ to $1.1 \times 10^{20} \mathrm{~cm}^{-3}$ at $-125 \mathrm{~V}$ after which only a gradual increase to $1.6 \mathrm{x}$ $10^{20} \mathrm{~cm}^{-3}$ at the highest bias. The peak-to-peak linewidth, Fig. 5(b), shows an initial increase in the linewidth reaching a maximum of $1.4 \mathrm{mT}$ at $-125 \mathrm{~V}$ bias, followed by a rapid decrease to $0.7 \mathrm{mT}$ and then a decrease to $0.56 \mathrm{mT}$ at $-265 \mathrm{~V}$ bias. At these higher biases the lineshape is Lorentzian. Finally, the Tauc gap falls rapidly from $2.6 \mathrm{eV}(-50 \mathrm{~V}$ bias) to $1.3 \mathrm{eV}(-125 \mathrm{~V})$ and then falls gradually to about $1.1 \mathrm{eV}$ at the highest biases.

From these results it is clear that there are two regimes, above and below $-125 \mathrm{~V}$ bias reflecting the change from PAC to DLC films. It is therefore convenient to discuss the results from the two regimes separately. For the sample deposited at $-125 \mathrm{~V}$ bias the measured spin density is $1.1 \times 10^{20} \mathrm{~cm}^{-3}$ and observed linewidth is $1.4 \mathrm{mT}$. The lineshape is Lorentzian which indicates a homogenous broadening mechanism, such as a dipole-dipole interaction. For a magnetically dilute material with $\mathrm{C}$ related centers the dipolar contribution to the linewidth $\Delta \mathrm{B}_{\mathrm{pp}}{ }^{\text {dipolar }}$ (mT), can be related (Barklie et al., 2000) to the spin density $\left(\mathrm{cm}^{-3}\right)$ via

$$
\Delta B_{p p}{ }^{\text {dipolar }}=8.1 \times 10^{-21} N_{s} .
$$


Using the measured value of $\mathrm{N}_{\mathrm{s}}$ determined for this film Eq. (2) gives a predicted linewidth of 0.9 $\mathrm{mT}$. This is slightly lower than the measured value of $1.4 \mathrm{mT}$ but indicates that the principal source of broadening is most likely to be dipolar broadening. At higher biases the spin density gradually increases but there is a reduction in the observed linewidth, in contrast to what that would be predicted by equation 2. This behaviour has been observed previously and was attributed to motional effects associated with the rapid exchange of spins as the $\mathrm{sp}^{2}$ cluster increases in size. This explanation also explains the narrowing of the Tauc gap since the size of the gap is related to the size of the cluster. The presence of motional effects can also be used to explain the similarity of the threshold fields measured for the DLC films. All these films will have a high $\mathrm{sp}^{2}$ content and the consequent overlap of the clusters will result in electron delocalisation and/or enhanced hopping between the clusters - Fig. 6(a). We regard this as an effective improvement in the connectivity between the clusters. Application of an external electric field results in extraction of electrons from the film surface. Replenishment of the emitted electrons to the surface layer is readily accomplished due to the good connectivity between the clusters. In this way emission from these films can be characterised as a 'front surface' type emission and since the paramagnetic $\mathrm{sp}^{2}$ clusters are believed to be located at the Fermi level the emission can be analysed within the Fowler-Nordheim theory.

In this way the FE is determined by the properties of the film near to the front surface. It is known from atomic force microscope images of DLC films that they are atomically flat with a typical rms roughness of less than $1 \mathrm{~nm}$. If a FN emission process is present then it is necessary to explain the large enhancement factors, typically several hundred, required to lower a barrier by 4 eV (Carey et al. 2001a). Since there are no surface features to aid the geometric enhancement of the electric field, we have examined the possibility of a non-geometric internal enhancement mechanism. The results of a high resolution scanning tunneling microscope (STM) study of the DLC film deposited at $-265 \mathrm{~V}$ are presented in Fig. 7. It is apparent that the conductivity of the film is not uniform with a number of localised high conductivity regions of a few $\mathrm{nm}$ in dimension 
surrounded by a more insulating matrix. The STM results can be interpreted as resulting from a series of closely spaced localised conductive $\mathrm{sp}^{2}$ regions which, under the action of the applied field electrons may hop from one spatially localised cluster to another. In the continuous limit this would produce a conducting channel or filament which can extend through the bulk of the film. Field enhancement due to a free standing 'protrusion' of length $h$ and radius $r$ can be taken to a first approximation as $h / r$. From the STM measurement if $r$ is taken to be $2 \mathrm{~nm}$ and a value of $\beta$ of 200 is inferred from the analysis of the $I-V$ characteristic, then $h$ must be about $200 \mathrm{~nm}$. High resolution electron microscopy studies of $a$-C films have never revealed such a large well defined object leading us to conclude that an alternative enhancement mechanism is present.

We propose the enhancement is due to the different intrinsic dielectric and conductive properties of nanometer sized $\mathrm{sp}^{2}$ clusters embedded in the surrounding $\mathrm{sp}^{3}$ insulating matrix. It is well known that the electric field near a single conductive dielectric sphere in a insulating matrix, is increased by a factor of up to 2 due to the dielectric mismatch between the sphere and the matrix (Griffiths, 1999). Chaumet and Dufour showed that the presence of two closely spaced spheres with the conductivity of gold, an enhancement of the electric field by a factor of 56 is possible if the spheres have a separation of $5 \mathrm{~nm}$ (Chaumet and Dufour, 1998). This enhancement increases as the separation between the sphere is reduced reaching a factor of 400 for a sphere-sphere separation of $1 \mathrm{~nm}$. Although the conductivity of the $\mathrm{sp}^{2}$ clusters will not be as high as Au, these electrostatic field distribution calculations demonstrate that high enhancement factors can be obtained by considering the effects of just two conductive spheres near the surface.

In this manner the emission process for DLC films can be explained as follows: the high density of defects will act to localise and attract the field lines from the anode to a thin region near the surface of the film. High field enhancement factors are present if two (or more) $\mathrm{sp}^{2}$ conductive clusters are nearby. Once the electrons are emitted from the clusters near the surface of the film, they can be replaced, by electrons from clusters deeper within the film. Such a description aids us in 
explaining the spotty non-uniform nature of the emission across the surface of the film in which the local arrangement of the clusters below the surface is important. This concept of dielectric inhomogeneity has also been applied to explain the field emission from boron-doped $\mathrm{Si}$ nanoparticle chains (Tang et al., 2001), amorphous $\mathrm{CN}_{\mathrm{x}}$ films prepared by pulsed laser deposition (Fogarassy et al., 2002), laser crystallised $a$-Si:H (Tang et al., 2002) as well as to ion beam synthesized SiC layers (Tsang et al., 2002).

In the other bias regime, the results presented in Fig. 5 show that reducing the self bias to $-90 \mathrm{~V}$ results in a reduction in the spin density by about two orders of magnitude and a rapid rise in the Tauc gap. The rise in the Tauc gap indicates a transition to a PAC film with a high $\mathrm{C}-\mathrm{H}$ bonding fraction and a lower concentration of isolated $\mathrm{sp}^{2}$ clusters. At low biases a low concentration of small isolated $\mathrm{sp}^{2}$ clusters is present. Initially, the number of $\mathrm{C}$ atoms in each cluster is small (as inferred from the large Tauc gap) but the greater energy available at higher biases allows the formation of a greater number of larger, but still isolated, clusters. This results in a reduction of the Tauc gap and an increasing spin density, though the electron delocalisation in the cluster and/or hopping between clusters is kept to a minimum. This leads to a poorer cluster-cluster interaction and explains why $\mathrm{E}_{\mathrm{th}}$ is higher at $-90 \mathrm{~V}$ bias when compared with at films deposited the higher biases. The subsequent drop in $\mathrm{E}_{\mathrm{th}}$ for the film at the lowest biases indicates that another emission mechanism is also present in these low defect polymeric wide energy gap films and is attributed to the transition to a back contract control mechanism as described earlier. In the description of the model above we can see the change from the front surface/internal controlled mechanism found in DLC to the back controlled mechanism proposed for PAC films. This demonstrates that there is more than one FE mechanism present and why care should be exercised in taking an explanation appropriate to one cathode system and applying it to another.

In summary, we believe that the field emission from $a-\mathrm{C}: \mathrm{H}$ films can be explained in terms of the number and size of $\mathrm{sp}^{2}$ clusters lying at or near to the Fermi level and it is important that the 
clusters be large enough for efficient connectivity between clusters. In this way the $a-\mathrm{C}: \mathrm{H}$ films deposited under different conditions will consist of a matrix of $\mathrm{sp}^{3} \mathrm{C}$ with regions of varying $\mathrm{sp}^{2}$ cluster concentration and size. Since these cluster will have different dielectric constants, the application of the external field will result in local field enhancements around the clusters and will aid in the emission of electrons. This model can also explain the non-uniformity of emission across the surface of the film in terms of different local concentrations of $\mathrm{sp}^{2}$ clusters through the film which extend from the surface.

\section{Controlling Inhomogeneity to Nanotip Cathodes}

It is apparent from the previous discussion that the size and concentration of the $\mathrm{sp}^{2}$ clusters and dielectric inhomogeneity, is important. It is possible to improve the FE characteristics by post deposition processing of the cathode which changes dielectric inhomogeneity. Several groups have reported that in the $a-\mathrm{C}$ films, in particular, that an initial conditioning treatment of the film is often required before the onset of stable emission is observed (Carey and Silva, 2001b, Missert et al., 1997). It was further noted that this conditioning of the cathode can be performed by cycling the voltage and that after several such cycles the values of the threshold fields tend to converge. It is also possible to condition the cathode though the intentional stressing either over large areas or using a STM tip. By using high resolution spatially-resolved electron energy loss spectroscopy (EELS) it was found that the predominant bonding configuration changes from predominately $\mathrm{sp}^{3}$ to $\mathrm{sp}^{2} \mathrm{C}$ coordinated (Missert et al., 1997). The net effect of the conditioning or the current stressing treatments is to generate conductive $\mathrm{sp}^{2}$ rich areas either in the form of filaments or clusters through localised Joule heating. A similar increase in the bulk conductivity of an $a$-C film has been reported through the controlled use of ion beams (Khan et al., 2001).

It is possible to extend this concept of dielectric inhomogeneity from the 'intrinsic', in

which the inhomogeneity is brought about by the $\mathrm{sp}^{2}$ clusters in the insulating $\mathrm{sp}^{3} \mathrm{C}$ matrix, to the 
intentional incorporation of different materials into a supporting matrix to form a composite emitter. One such study to make use of 'extrinsic' dielectric inhomogeneity is the incorporation of conducting inks made from conducting micron sized particles in an insulating matrix is by UK company Printable Field Emitters Ltd. (Burden et al., 2001 b). The structure of these nearly flat composite emitters (Fig. 6b) can be viewed as consisting of metal-insulator-metal-insulatingvacuum (MIMIV). The application of an electric field results in electron transport from the back metal across the MIM path and then emission from a MIV region near the surface. Field enhancement at the tips of the conducting particles aids in the emission process and therefore control of the particle size distributions is important. One of the immediate advantages of this arrangement is that the presence of the insulating layer also acts to protect the cathode material from poor vacuum conditions and contamination. Furthermore the cathode system discussed has the added advantage of being compatible with screen printing or ink-jet printing technology allowing for broad-area deposition. Both diode and triode arrangements have been processed and the company have recently reported the construction of a 320 x 240 pixel demonstrator equivalent to a 5.7 inch quarter VGA monochrome display.

All these types of cathode materials have some design features in common such as an ability to deposited over large areas, using conventional non-lithographic processes such as plasma deposition or screen printing. Optimisation of the cathode material can be performed by selecting the correct film thickness, deposition conditions and post deposition treatments or the size of particulate inclusions. Whilst the fabrication of large area Spindt tip based arrays have well documented problems, tip based structures with (relatively) easy deposition and processing have received renewed interest with the production of carbon nanotubes (CNTs). Whilst the growth and FE characteristics of CNTs have been reviewed elsewhere (Bonard et al. 2001a), the key aspect for these materials is the geometric enhancement factor associated with the nanotube's aspect ratio. Electron emission from an individual nanotube has been observed, however, for the practicality of a 
display, films or mats of nanotubes are required as shown in Fig. 8. Placing nanotubes in proximity to one another results in a reduction of the enhancement factor due to the screening of the applied field. In addition, there will be a distribution in nanotube dimensions (length and radius) as well as local environments with the result that there will be a distribution of field enhancement factors. This will result in different nanotubes experiencing different local electric fields for a given applied field. As a consequence raising the applied field will result in the nanotubes with the highest enhancement factors emitting at the lowest applied field. For an ideal display with uniform emission it is desirable that as many of the emitting sites to turn on at the same field. In addition emission sites with low turn on fields will tend to carry most of the emission current, resulting in high current densities. This can result in substantial Joule heating at the end of the tube, though the thermal behaviour depends on the preparation of the tube. At high tube temperatures the removal of absorbed atoms or any amorphous carbon layer that can grown during chemical vapour deposition may occur. High current densities can also result in the tube tip rounding and eventually burning out. As the applied field increases other tubes may turn on giving a more uniform emission density. Intentional tube burn out is a way of conditioning the cathode to ensure uniform emission. To date Samsung have fabricated fully vacuum-sealed 5 in. diagonal CNT FED. The onset gate electrode voltage for emission was about $60 \mathrm{~V}$ and the luminance as high as $510 \mathrm{~cd} / \mathrm{m}^{2}$ was reported under the application of $100 \mathrm{~V}$ and $1.5 \mathrm{kV}$ to gate electrode and anode, respectively (Chung et al., 2002).

Uniform emission is also important for the possible use of carbon nanotubes in lighting. The possibility of using field emission from CNTs grown on cylindrical diodes in which multiwall CNTs were grown on a metallic wire as a source of lighting has been demonstrated (Bonard et al., 2001b). The anode is an ITO glass tube with a phosphor layer deposited on the inner surface. Application of $5.4 \mathrm{kV}$ to the anode yielded an emitted current density of $0.5 \mathrm{~mA} / \mathrm{cm}^{2}$ on the cathode and $0.06 \mathrm{~mA} / \mathrm{cm}^{2}$ on the anode. Using a photometer a luminance of $10^{5} \mathrm{~cd} / \mathrm{m}^{2}$ was observed, a value that can be compared with a commercial mercury containing fluorescence tube of $1.1 \times 10^{5}$ 
$\mathrm{cd} / \mathrm{m}^{2}$. Whilst the power used was an order of magnitude larger than that for the commercial available system, this proof of concept does demonstrate that field emission from CNTs may find an application as a sustainable source of environmentally friendly lightening. Whilst carbon nanotubes have attracted the most attention electron emission has also been observed from a wide range of nanotube materials including $\mathrm{SiC}$ nanowires (Wong et al., 1999), $\mathrm{Cu}_{2} \mathrm{~S}$ nanowires (Chen et al., 2002), W nanowires (Lee et al., 2002) and nanobelts of $\mathrm{MoO}_{3}$ (Li et al., 2002).

\section{Outlook for the future}

Finally I wish to highlight one way to combine many of the advantageous properties discussed above. An ideal broad area field emitting material, regardless of the details of the mechanism, should

(i) be able to be deposited over large area using technologically mature and relatively inexpensive techniques such as chemical vapour deposition or screen printing.

(ii) The value of the threshold field should be as reproducible as possible and to limit the spread in the drive voltages, any hysteresis in the current-voltage $(I-V)$ characteristics should be kept to a minimum.

(iii) There should be good control over the uniformity of emission either by controlling the size distribution of the emitter sites such as CNTs or embedded ink particles by current conditioning the samples if necessary.

(iv) Finally a way of protecting the cathode from residual gas atoms and poor vacuum should be envisaged.

In this way recent studies into carbon nanotube composites may have all the advantages discussed above. Shown in Fig. 8 is a scanning electron micrograph of carbon nanotubes embedded in a polystyrene matrix. Poa and colleagues (Poa et al., 2002) have shown that CNTs, both undoped and B doped MWCNTs grown by arc discharge method, embedded in a polystyrene matrix exhibit 
excellent field emission properties with threshold fields of $1.6 \mathrm{~V} / \mu \mathrm{m}$ and $2.6 \mathrm{~V} / \mu \mathrm{m}$ respectively, for a nanotube concentration of $12.5 \%$. Increasing the nanotube concentration resulted in higher threshold fields, a behaviour attributed to the screening effects discussed above. Efforts have also been made to investigate possible alternatives to the high temperature $\left(>650^{\circ} \mathrm{C}\right) \mathrm{CVD}$ deposition of CNTs. Recently there has been a report of the growth of carbon nanofibers at room and low temperatures temperature (Boskovic et al., 2002). This opens up the possibility of growing nanotube displays on glass or plastic substrates. Preliminary FE emission measurements have shown that emission is possible to obtain emission at low applied fields (Smith, 2003).

In conclusion it has become apparent that flat films of amorphous carbon thin films, conducting particles in an insulating matrix and carbon nanotubes both as mats or embedded in polystyrene matrix all show some commonality in their field emission behaviour. All these types of cathode can be deposited over large area using conventional deposition methods. All have been well characterised in terms of material and electrical properties but it is only by looking at the cathodes on a nanometer scale does the similarity between the different systems become apparent. It is hoped that in parallel to the production of working displays a fuller understanding of the field emission mechanisms of these materials can be developed.

\section{Acknowledgements}

I would like to acknowledge the contribution, advice and guidance over the years of Professor S.R.P. Silva as well all the other members of the Large Area Electronics Group in the Advanced Technology Institute of the University of Surrey. The author is grateful to the EPSRC for an Advanced Research Fellowship GR/R76288. 
Published in Phil. Trans. Roy. Soc. A 361, 2891 (2003)

\section{References}

Amaratunga GAJ and Silva SRP 1996 Nitrogen containing hydrogenated amorphous carbon for thin-film field emission cathodes. Appl. Phys. Lett. 68, 2529 - 2531.

Barklie RC, Collins M and Silva SRP 2000 EPR linewidth variation, spin relaxation times, and exchange in amorphous hydrogenated carbon. Phys. Rev. B 61, $3546-3554$.

Bonard JM, Kind H, Stockli T, and Nilsson LA 2001 Field emission from carbon nanotubes: the first five years. Solid State Electr. 45, 893 - 914.

Bonard JM, Stockli T, Noury O and Chatelain A 2001, Field emission from cylindrical carbon nanotube cathodes: Possibilities for luminescent tubes. Appl. Phys. Lett. 78, 2775 - 2777.

Boskovic BO, Stolojan V, Khan RUA, Haq S and Silva SRP 2002 Large-area synthesis of carbon nanofibers at toom temperature. Nature Materials 1, 165 - 167.

Burden AP 2001a Materials for field emission displays. Int. Mat. Rev 46, 213 - 231.

Burden AP, Bishop HE, Brierley M, Friday JM, Hood C, Jones PGA, Khazov AY, Lee W, Riggs RJ, Shaw VL, Taylor W, Tuck RA 2001b Incorporating consumer-priced field-emitting inks into arrays of triode devices. Solid State Electr. 45, 987 - 996.

Carey JD, Forrest RD, Khan RUA, and Silva SRP 2000 Influence of $\mathrm{sp}^{2}$ clusters on the field emission properties of amorphous carbon thin films. Appl. Phys. Lett. 77, 2006 - 2008.

Carey JD, Forrest RD, and Silva SRP 2001a Origin of electric field enhancement in field emission from amorphous carbon thin films. Appl. Phys. Lett. 78, 2339 - 2341.

Carey JD and Silva SRP 2001b Conditioning of hydrogenated amorphous carbon thin films for field emission via current stressing. Appl. Phys. Lett. 78, $347-349$.

Chaumet PC and Dufour JP 1998 Electric potential and field between two different spheres. J. Electrost. 43, 145 - 159. Chen J, Deng SZ, and Xu NS 2002 Field emission from crystalline copper sulphide nanowire arrays. Appl. Phys. Lett., 80, $3620-2622$.

Chung DS, Park SH, Lee HW, Choi JH, Cha SN, Kim JW, Jang JE, Min KW, Cho SH, Yoon MJ, Lee JS, Lee CK, Yoo JH, Kim JM, Jung JE, Jin YW, Park YJ, You JB 2002 Carbon nanotube electron emitters with a gated structure using backside exposure processes. Appl. Phys. Lett. 80, 4045 - 4047.

Fogarassy E, Szorenyi T, Antoni F, Stoquert JP, Pirio G, Olivier J, Legagneux P, Boher P, Pons-Y-Moll O 2002 Influence of the nitrogen content on the field emission properties of $a-\mathrm{CN}_{\mathrm{x}}$ films prepared by pulsed laser deposition. Appl. Surf. Sci. 197, 316-320. 
Published in Phil. Trans. Roy. Soc. A 361, 2891 (2003)

Forrest RD, Burden AP, Silva SRP, Cheah LK and Shi X 1998 A study of electron field emission as a function of film thickness from amorphous carbon films. Appl. Phys. Lett. 73, $3784-3786$.

Fowler RH and Nordheim L 1928 Electron emission in intense electric fields. Proc. Roy. Soc. Lond., Ser A 119, 173 181.

Funaki Y and Mochizuki Y 1999 Flat panel display market to reach 10 trillion yen by 2010. Flat panel display yearbook, InterLingua, Redondo Beach, California, p. 75.

Griffiths DJ 1999 Introduction to electrodynamics, Prentice-Hall, New Jersey, p. 146.

Ilie A, Ferrari A.C, Yagi T, and Robertson J 2000 Effect of $\mathrm{sp}^{2}$-phase nanostructure on field emission from amorphous carbons. Appl. Phys. Lett. 76, 2627 - 2629.

Khan RUA, Carey JD, Silva SRP, Jones BJ and Barklie RC 2001 Electron delocalisation in amorphous carbon by ion implantation. Phys. Rev. B 63, art. no. 121201.

Lee Y-H, Choi C-H, Jang Y-T, Kim E-K, and Ju B-K 2002, Tungsten nanowires and their field electron emission properties. Appl. Phys. Lett. 81, $745-747$.

Li YB, Bando Y, Golberg D, and Kurashima K 2002 Field emission from $\mathrm{MoO}_{3}$ nanobelts. Appl. Phys. Lett. 81, 5048 5050.

Missert N, Friedmann TA, Sullivan JP, and Copeland RG 1997 Characterization of electron emission from planar amorphous carbon thin films using in situ scanning electron microscopy. Appl. Phys. Lett. 70, 1995 - 1997.

Poa CH, Silva SRP, Watts PCP, Hsu KW, Kroto HW and Walton DRM 2002 Field emission from nonaligned carbon nanotubes embedded in a polystyrene matrix. Appl. Phys. Lett. 80, $3189-3191$.

Samuel IDW 2000 Polymer electronics. Philos. Trans Roy Soc A 358, 193 - 210.

Silva SRP, Carey JD, Khan RUA, Gerstner EG and Anguita JV 2002 Amorphous carbon thin films chapter 9, volume 4, in Handbook of Thin Films, ed. H. S. Nalwa (Academic Press, New York), 403 - 506.

Smith RC, Poa, CH, Cox DC, Carey JD, and Silva SRP 2003, unpublished.

Tanaka K 1999 Amorphous silicon, John Wiley \& Sons, Inc. (New York).

Tang YF, Silva SRP, Boskovic BO, Shannon JM, and Rose MJ 2002 Electron field emission from excimer laser crystallized amorphous silicon. Appl. Phys. Lett. 80, $4154-4156$.

Tang YH, Sun XH, Au FCK, Liao LS, Peng HY, Lee CS, Lee ST, and Sham TK 2001 Microstructure and fieldemission characteristics of boron-doped Si nanoparticle chains. Appl. Phys. Lett. 79, 1673 - 1675.

Tsang WM, Wong SP, and Lindner JKN 2002 Origins of field enhancement in electron field emission from ion beam synthesized SiC layers. Appl. Phys. Lett. 81, $3942-2944$. 
Published in Phil. Trans. Roy. Soc. A 361, 2891 (2003)

Wong KW, XT Zhou, Au FCK, Lai KL, Lee CS, and Lee ST 1999 Field-emission characteristics of SiC nanowires prepared by chemical-vapor deposition. Appl. Phys. Lett. 75, $2918-2920$.

Wood RW 1897 A New form of cathode discharge and the production of X-rays together with some notes on diffraction. Phys. Rev. 5, $1-10$.

Zhu W 2001 Vacuum Microelectronics, John Wiley \& Sons, Inc. (New York). 
Published in Phil. Trans. Roy. Soc. A 361, 2891 (2003)

\section{Figure captions}

Figure 1. Different current and emerging display technologies. The key to the figure is: CRT - Cathode ray tube, DMD - Digital mirror device, LCD - Liquid crystal display, FPD - Flat panel display, TFT - Thin film transistor, MOS - Metal-oxide-semiconductor, MIM - Metal-insulator-metal, FLC - Ferroelectric crystal, STN - Super twisted nematic liquid crystal, PDLC - Polymer dispersed liquid crystal, PSCT - Polymer stabilized cholesteric texture.

Figure 2. Current and emerging technologies for different screen size diagonals. Adapted with permission (Burden 2001a).

Figure 3. Illustration of the different physical processes that can occur form a metal surface upon application of an electric field.

Figure 4. (a) Schematic of a Spindt tip field emitter. The tip material is usually metal or Si and emission is accomplished by applying a voltage between the gate and the tip. (b) Schematic phase diagram showing the different types of amorphous carbon film that are possible DLC - Diamond like carbon, TAC - tetrahedral amorphous carbon, PAC - polymeric amorphous carbon, GAC - graphitic like amorphous carbon.

Figure 5. Variation of (a) threshold electric field (!) and spin density (-) and (b) peak-to-peak line width (,) and Tauc gap (8) as a function of deposition self bias.

Figure 6. Schematic showing (a) the transport of electrons between $\mathrm{sp}^{2}$ clusters embedded in $\mathrm{sp}^{3}$ rich matrix (b) the emission of electrons from the MIMIV structure found in the conducting particles embedded in a insulating resin based upon the design of Printable Field Emitters Ltd.

Figure 7. High resolution scanning tunnelling microscope image of a DLC film showing non uniformity of the current. The image shows current inhomngoneto4es in the region of few nanometers. 
Published in Phil. Trans. Roy. Soc. A 361, 2891 (2003)

Figure 8. Scanning electron microscope image of carbon nanotubes embedded in a polystyrene matrix. Image courtesy of Dr D Cox, University of Surrey. 


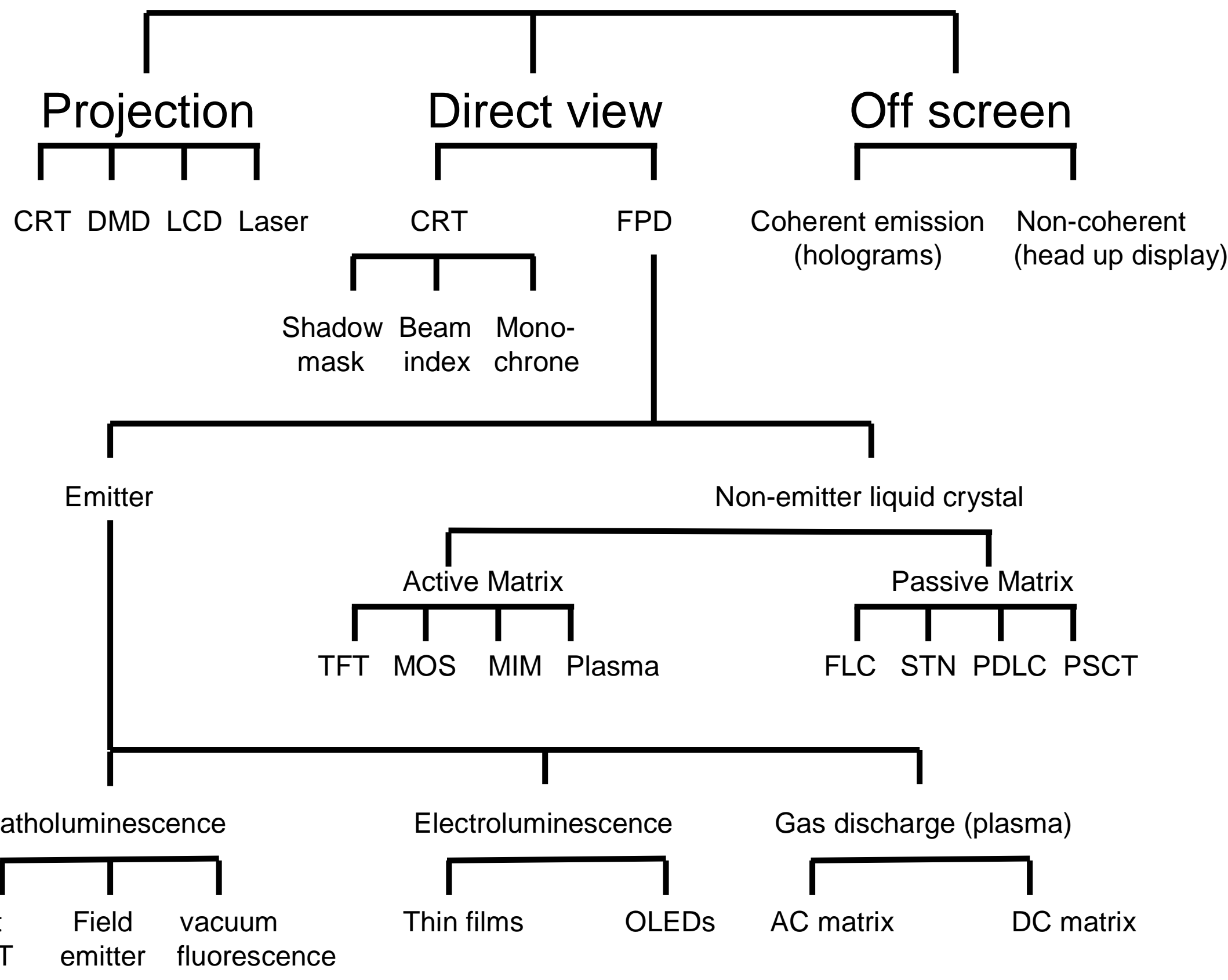




\section{Existing technology}

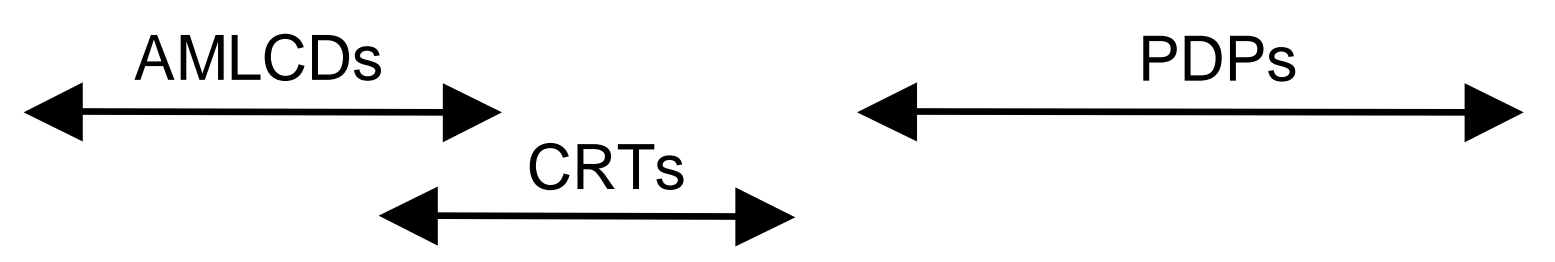

Spindt FEAs

$\begin{array}{lllllll}2.5 & 25 & 50 & 75 & 100 & 150 & \text { screen diagonal }(\mathrm{cm})\end{array}$
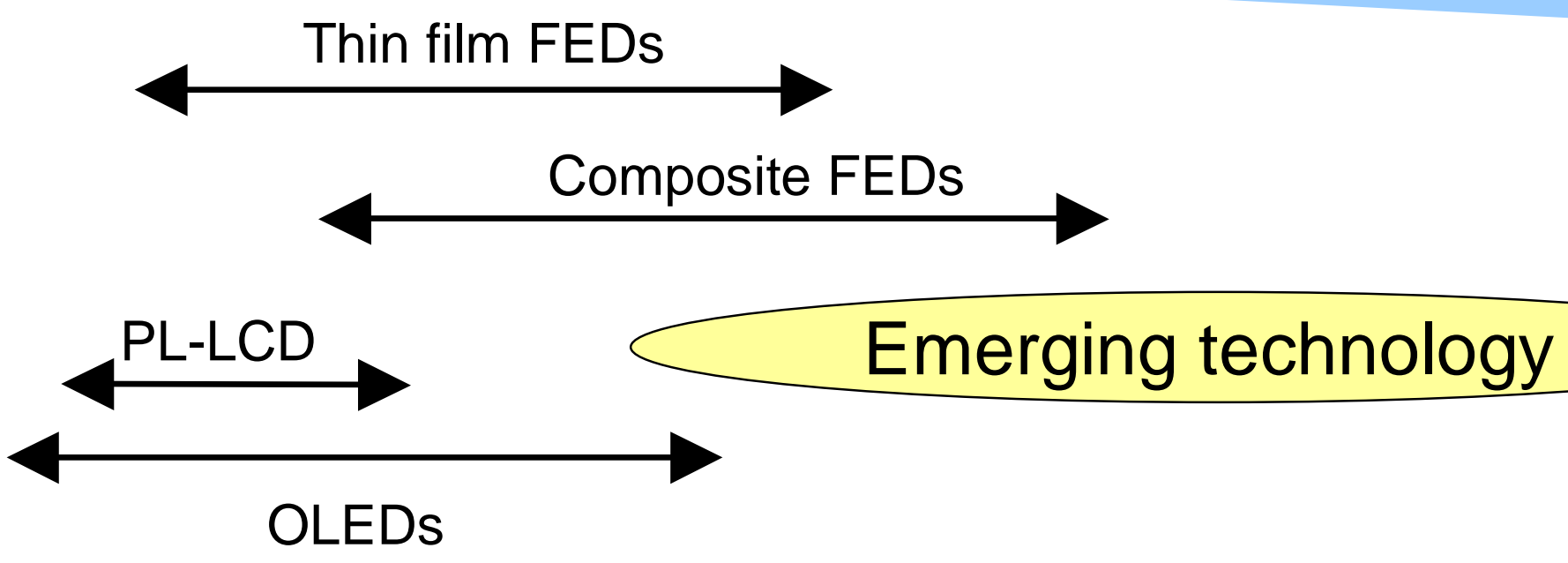
(a)

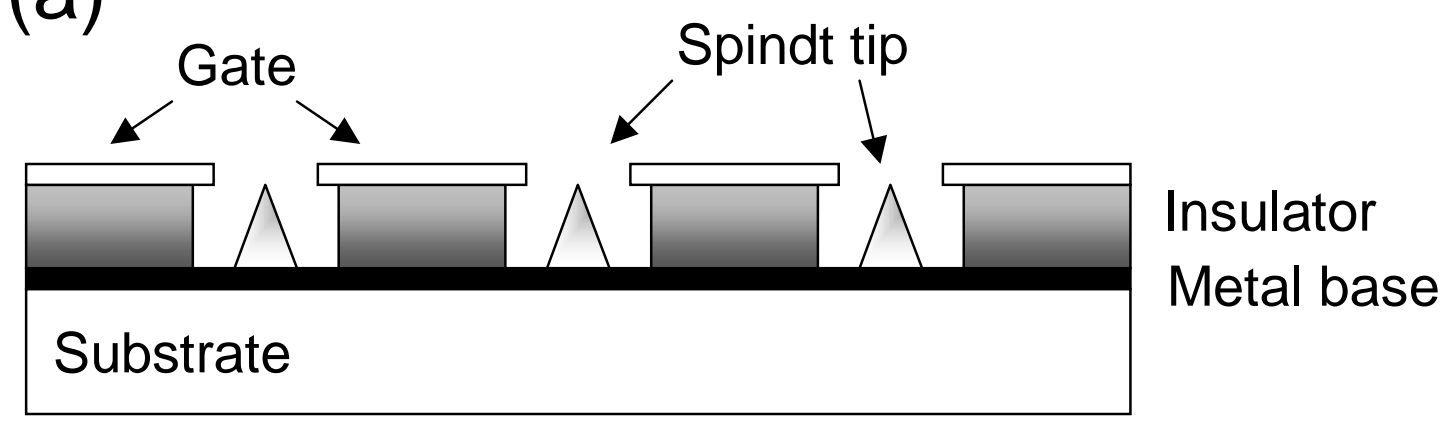

(b)

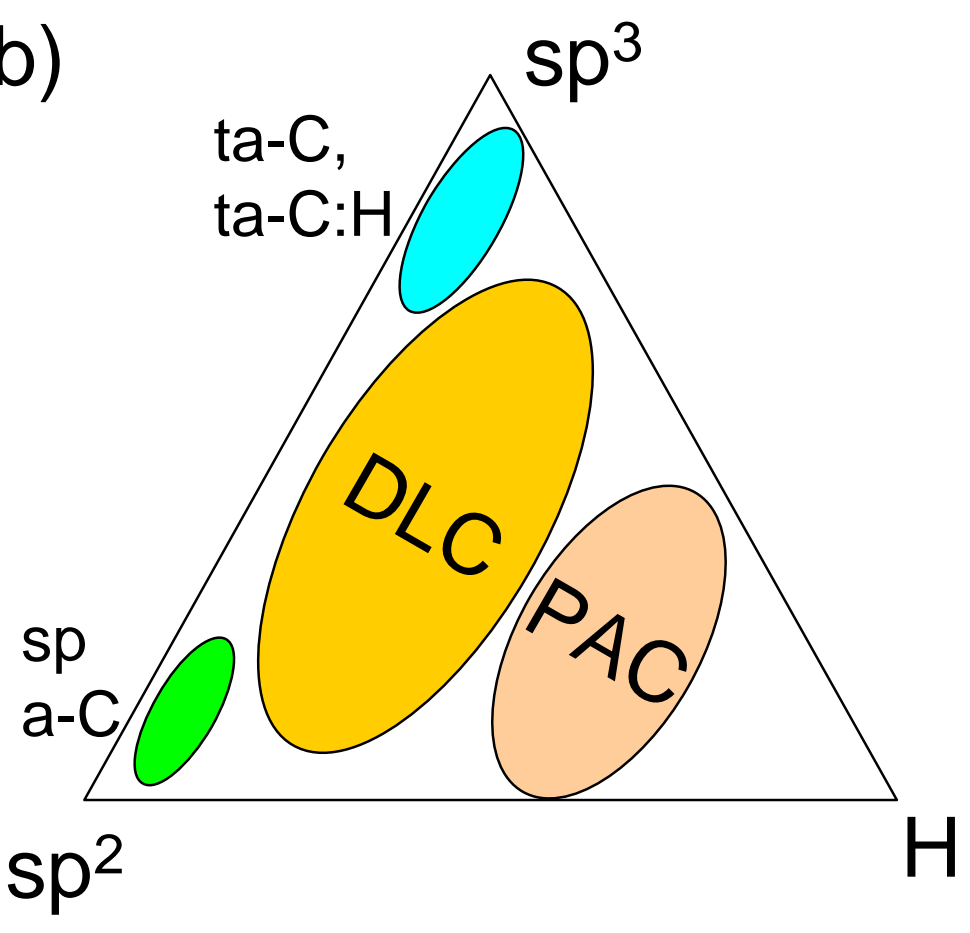




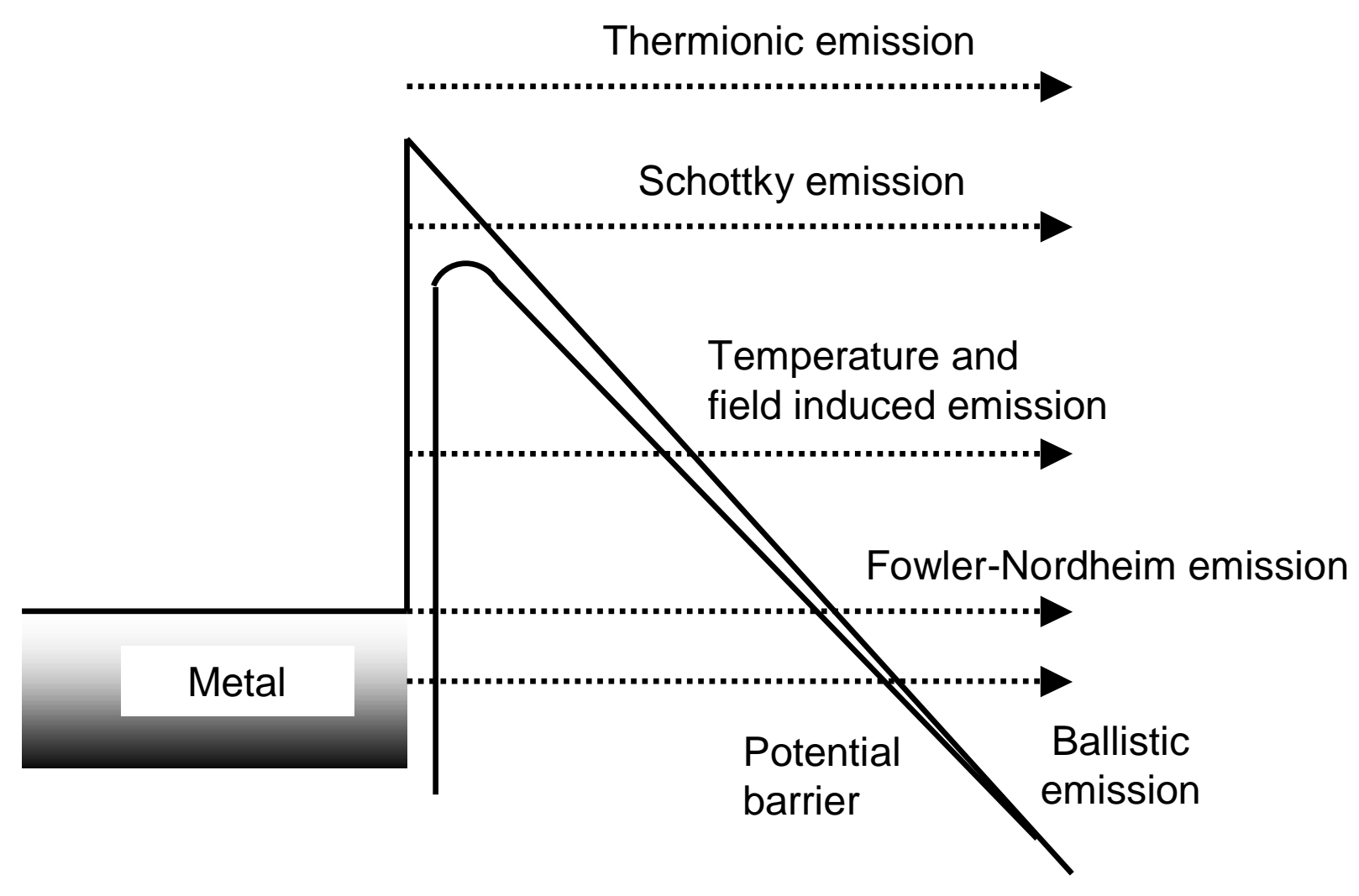




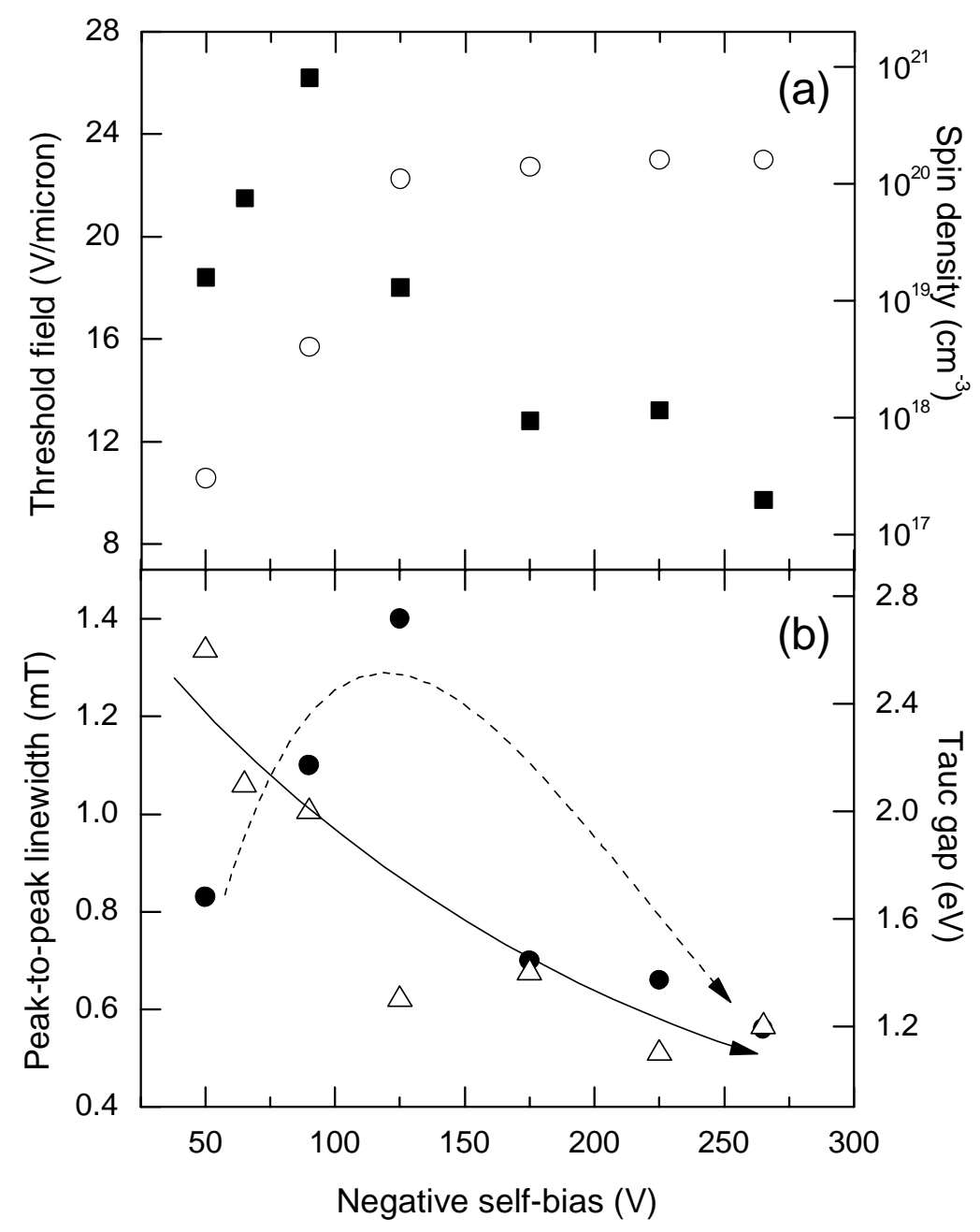

JD Carey Fig 5 


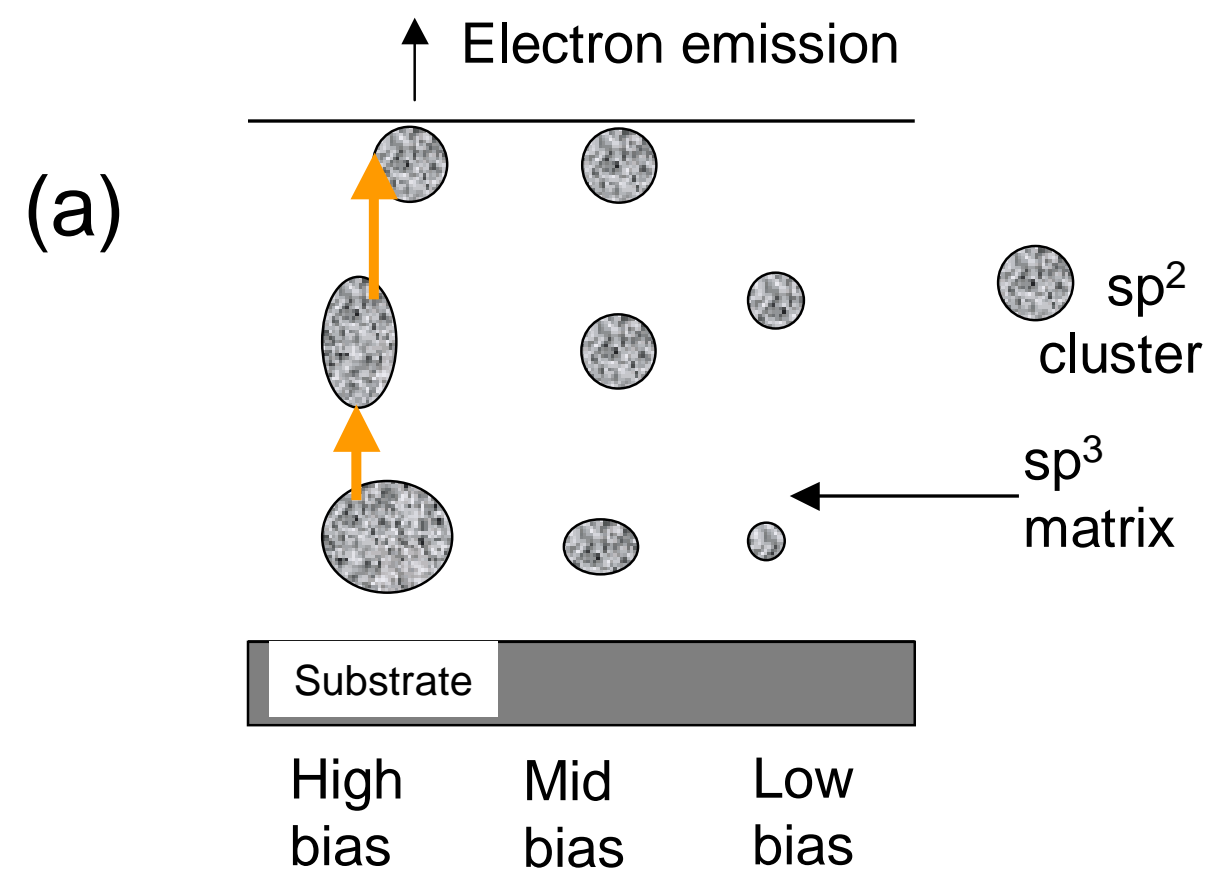

(b)

\section{Electron emission}

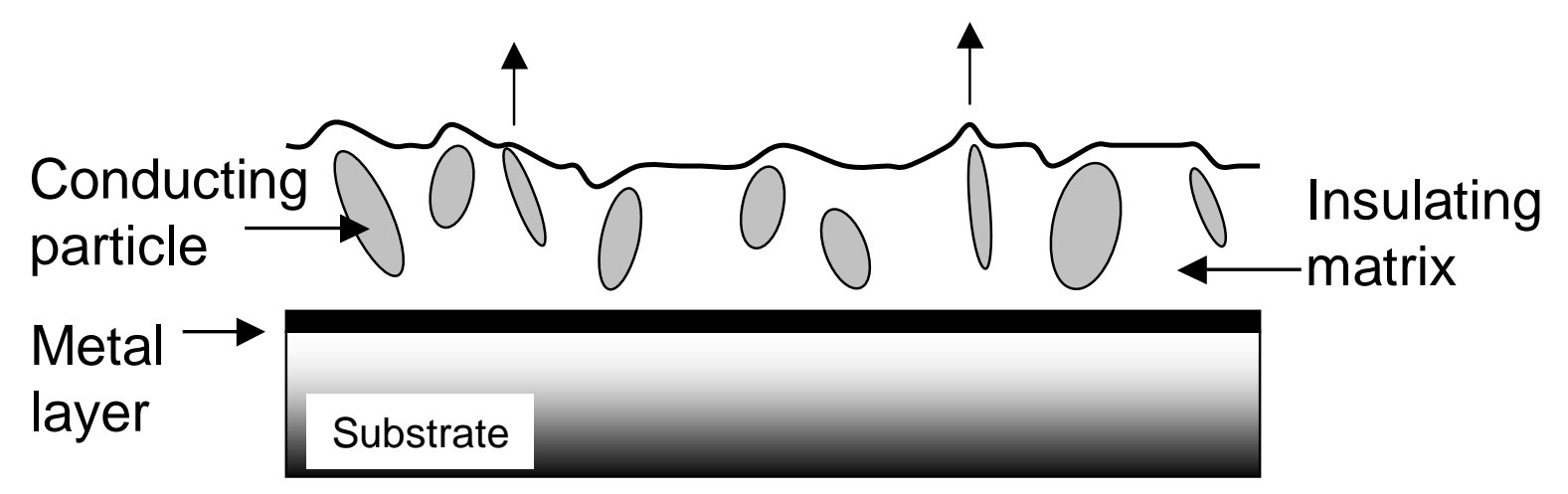




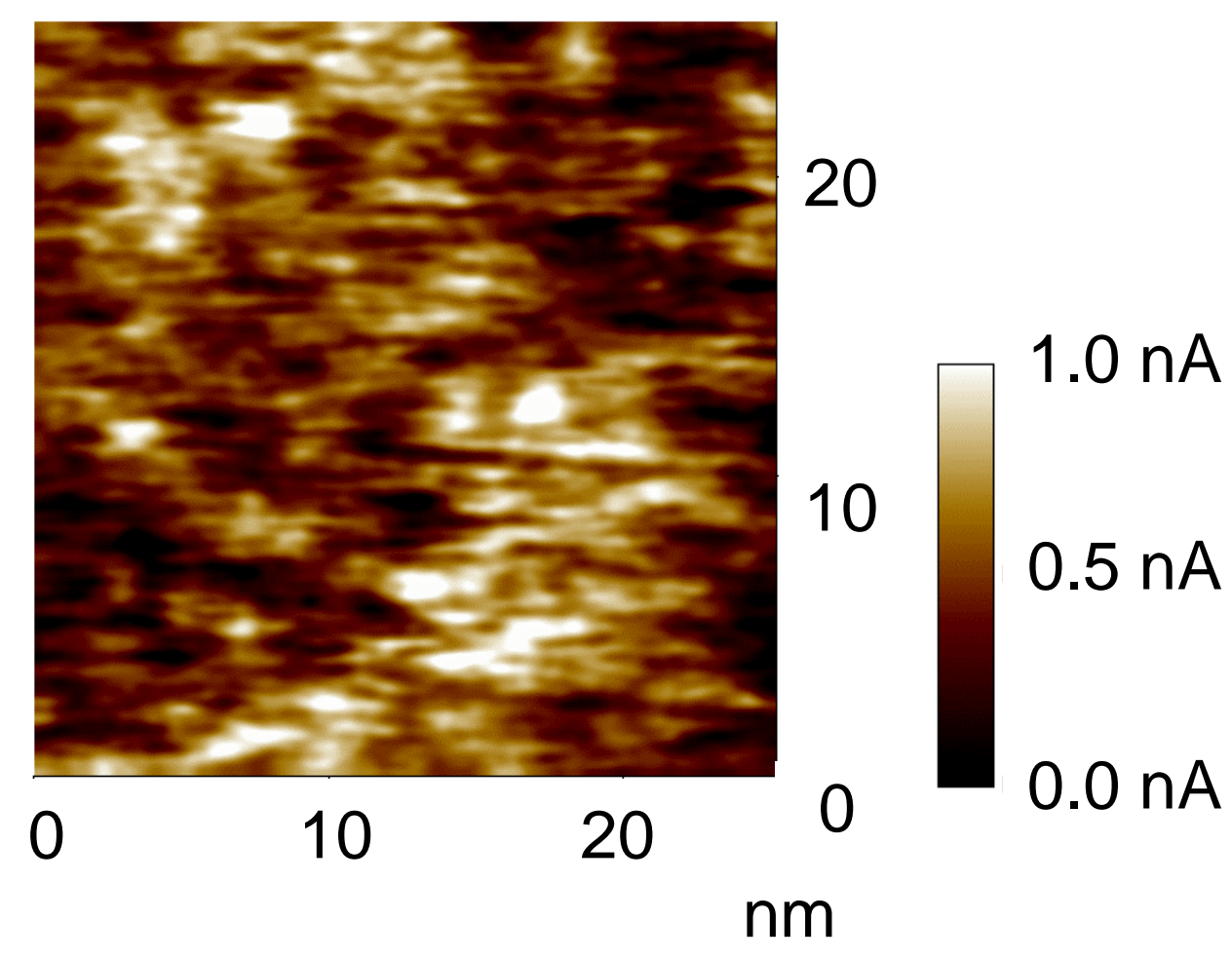

